\title{
Diffraction tomography for \\ multi-monostatic ground penetrating radar imaging \\ Final Project Report
}

D.O.E. Grant DE-FG02-93ER14392

Anthony J. Devaney and Ross W. Deming*

Department of Electrical and Computer Engineering

Northeastern University, Boston, MA 02115

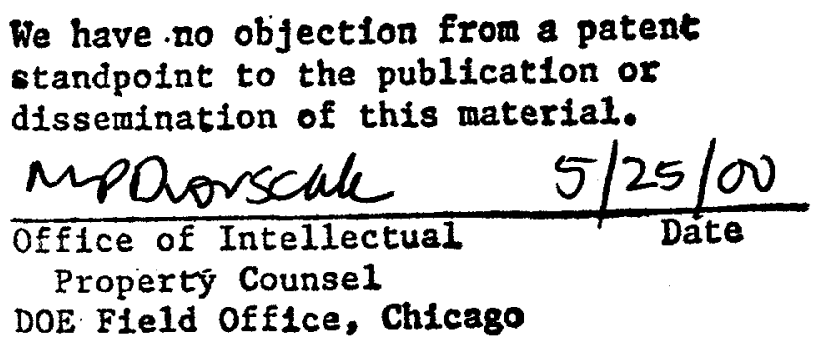

*This report represents research carried out by the author in the doctoral program at Northeastern University. 


\section{DISCLAIMER}

This report was prepared as an account of work sponsored by an agency of the United States Government. Neither the United States Government nor any agency thereof, nor any of their employees, make any warranty, express or implied, or assumes any legal liability or responsibility for the accuracy, completeness, or usefulness of any information, apparatus, product, or process disclosed, or represents that its use would not infringe privately owned rights. Reference herein to any specific commercial product, process, or service by trade name, trademark, manufacturer, or otherwise does not necessarily constitute or imply its endorsement, recommendation, or favoring by the United States Government or any agency thereof. The views and opinions of authors expressed herein do not necessarily state or reflect those of the United States Government or any agency thereof. 


\section{DISCLAIMER}

Portions of this document may be illegible in electronic image products. Images are produced from the best available original document. 


\begin{abstract}
This report documents work performed under DOE Grant DE-FG02-93ER14392 by the PI Professor Anthony J. Devaney and a graduate student Ross Deming. The project produced a number of publications, including Ross Deming's Ph.D. thesis. The publications directly resulting from work performed on the project were:
\end{abstract}

1. A. Witten, A. Schatzberg, and A.J. Devaney, "Vector Radar Wave Diffraction Tomography Maximum Likelihood Estimation," Journal of Environmental \& Engineering Geophysics 0, pp.91-104, 1996.

2. R. Deming and A.J. Devaney, "A Filtered Backpropagation Algorithm for GRP," Journal of Environmental \& Engineering Geophysics 0, pp.113-124, 1996.

3. R. Deming and A.J. Devaney, "Diffraction tomography for multi-monostatic ground penetrating radar imaging ," Inverse Problems 13, pp.29-45, 1997.

4. R. Deming, "New Tomographic Imaging Methods for Geophysical and Medical Applications,", Ph.D. thesis, Department of Electrical and Computer Engineering, Northeastern University, Boston, MA, September, 1996.

The current report summarizes much of the work reported in the third paper listed above (the Inverse Problems paper) which is the major contribution of the work performed in the grant. In particular, a generalized diffraction tomographic (DT) algorithm is derived for subsurface imaging from multi frequency multi-monostatic ground penetrating radar (GPR) data. The algorithm is based on the Born approximation for vector electromagnetic scattering and incorporates realistic near field models for the receiving and transmitting antennas. The forward scattering model is inverted analytically using the regularized pseudo inverse operator to yield an algorithm for imaging the underground region based on scattered field measurements at a set of receiving antennas. Whereas the usual inversion algorithms of DT require a lossless background medium and ideal point sources and receivers, the algorithm described here allows an attenuating background and arbitrary transmitting and receiving antennas. The algorithm places no restrictions on the radar frequency, and can thus include shallow imaging applications where the wavelengths are on the same order as the depth of buried objects of interest. Versions of the algorithm are given for both the 3 dimensional and the 2.5 dimensional cases. Results are given of computer simulations designed to test the algorithm. 


\section{Introduction.}

In this report we present a new approach for subsurface imaging from multi frequency multimonostatic data measurements obtained using ground penetrating radar (GPR) in a reflection geometry. The method employs a linear scattering model for electromagnetic wave fields based on the Born approximation, which is inverted analytically to yield an image of the subsurface based on scattered field measurements. Thus, our imaging algorithm is related to the well established method of diffraction tomography (DT), which is used in various forms for such applications as optical inverse scattering [15], medical ultrasonic imaging [6, 14], and geophysical imaging $[7,8,20,26,27,28,29]$. In contrast with many applications of DT $[6,7]$, however, in GPR imaging background losses are significant and evanescent wave field components are important since radar wavelengths are often times on the same order as the depth and size of underground objects of interest [22]. Therefore in this development, unlike the usual treatments of DT, we incorporate soil attenuation into the mathematical inversions, and include evanescent components to help combat the restrictions on image resolution imposed by the relatively low frequencies used. Moreover, we employ realistic near field models for the transmitting and receiving antennas, in contrast with most treatments that employ ideal point sources and receivers. Our method can be used with band limited pulses, and can include a constant spatial offset between the transmitter and receiver in each monostatic experiment.

Several authors have addressed, within the context of DT, the problem of inverse scattering using multi-monostatic field measurements. In [19] exact inversion formulas, within the Born approximation, are derived using broadband multi-monostatic measurements conducted on planar, spherical, and cylindrical surfaces. The authors use an ideal point source and receiver approximation, and assume the distance to scattering objects is much greater than a wavelength. This treatment requires transmitted pulses which are not band limited although the authors suggest Wiener filtering as a means to circumvent this restriction. An iterative, time domain, approach to the multi-monostatic imaging problem is suggested in [25] based on the Born approximation. The background medium is assumed to be lossless and nondispersive, and the ideal point source and receiver approximation is used. In [27] DT algorithms for GPR imaging are derived and tested on experimental data. The Born approximation is employed, as well as the approximations that the background is lossless and the distance between antennas and scatterers is much greater than a wavelength. A target detection algorithm is also given which can incorporate an attenuating background. Efficient DT imaging methods are described in [29] using multi frequency multi-monostatic data for both constant and vertically varying backgrounds. Inversion formulas are given using both the Born and the physical optics approximations, assuming a lossless background medium and ideal point sources and receivers. With these algorithms the final image is formed by coherent superposition of sub images from each frequency, whereas we show in this report that an optimum multi frequency algorithm must exploit the correlation between the data 
at different frequencies.

The approach employed in this report consists of several steps. We first define a vector electromagnetic forward scattering model based on the Born approximation, which incorporates near field characteristics of the transmitting and receiving antennas via the scattering matrix models developed by Kerns [13]. This model then yields a coupled set of integral equations relating the data at each excitation frequency to the sought after "object function" defining the complex index of refraction profile of the subsurface. After casting the integral equations in a mathematical operator formalism, we are then able to find the minimum $L^{2}$ norm solution to the object function by deriving and applying the regularized pseudo inverse operator $[2,17,21]$. The method is found to be similar to filtered backpropagation algorithms of DT described in the literature $[4,6,7]$, and like these algorithms it is quantitative, and is designed to yield a solution which is mathematically consistent. with the measured data. The inversion is fully analytic, and therefore relatively efficient to compute.

In Section 2 we derive the forward scattering model in detail, then in Section 3 we derive the regularized inversion of this model for the case when the scatterer is three dimensional and monostatic experiments are conducted over the $x y$ plane defining the ground surface. In Section 4 we modify the three dimensional method to the 2.5 dimensional case where the scatterer is invariant in the $\hat{\mathbf{y}}$ direction and experiments are conducted along the $\hat{\mathbf{x}}$ axis. The report includes computed results from synthetic data in Section 5 , as well as a discussion of future directions for the methodology of this report in Section 6. 


\section{Forward model for electromagnetic scattering.}

By probing the earth with electromagnetic wave fields we wish to estimate the electrical permittivity distribution in the underground region $z<0$ from scattered field measurements at the surface $z=0$ (where the orientation of the coordinate axes are illustrated in Fig. 1). The incident (probing) fields are generated by a ground penetrating radar (GPR) system operating in either a pulsed time domain or step frequency mode. Our scattering model is developed here in the frequency domain, related to the time domain through the standard Fourier and inverse Fourier transform definitions.

The GPR survey is assumed to consist of a number of monostatic experiments, each corresponding to a different location of the transmitting/receiving antenna on the ground surface, and each incorporating data collected over a band of frequencies $\omega$. In each experiment the scattered field results from the interaction of the incident field with inhomogeneities in the subsurface, described by the object function

$$
O(\mathbf{r}, \omega)=1-\frac{\epsilon(\mathbf{r}, \omega)}{\epsilon_{0}(\omega)}
$$

Here, $\mathbf{r}=(x, y, z)$ is the three dimensional spatial coordinate and $\epsilon(\mathbf{r}, \omega)=\epsilon^{\prime}(\mathbf{r}, \omega)+$ $i \sigma(\mathbf{r}, \omega) / \omega$ is the complex permittivity in the underground, i.e. the soil with the embedded inhomogeneities. The quantity $\epsilon^{\prime}(\mathbf{r}, \omega)$ is the real dielectric constant and $\sigma(\mathbf{r}, \omega)$ is the conductivity, while $\epsilon_{0}(\omega)$ is the complex permittivity of the homogeneous background soil medium. It is assumed that the magnetic permeability in the underground is equal to $\mu_{0}$ in a vacuum. Throughout the discussion, MKS units are used.

The Fourier amplitude of the electric field vector generated in any given experiment satisfies the well known Lippmann Schwinger equation $[3,11,16]$

$$
\begin{aligned}
\mathbf{E}(\mathbf{r}, \omega) & =\mathbf{E}_{i n c}(\mathbf{r}, \omega)+\mathbf{E}_{s c a t}(\mathbf{r}, \omega) \\
& =\mathbf{E}_{i n c}(\mathbf{r}, \omega)-k_{0}^{2}(\omega) \int d^{3} r^{\prime} \overline{\mathbf{G}}\left(\mathbf{r}-\mathbf{r}^{\prime}, \omega\right) \cdot \mathbf{E}\left(\mathbf{r}^{\prime}, \omega\right) O\left(\mathbf{r}^{\prime}, \omega\right),
\end{aligned}
$$

where $\mathbf{E}_{\text {inc }}(\mathbf{r}, \omega)$ is the incident field for the experiment, $\mathbf{E}_{\text {scat }}(\mathbf{r}, \omega)$ is the scattered field component of the electric field vector, $k_{0}(\omega)=\omega \sqrt{\epsilon_{0}(\omega) \mu_{0}}$ is the complex wavenumber of the homogeneous background soil medium, and the Green's Dyadic $\overline{\mathbf{G}}(\mathbf{r}, \omega)$ satisfies

$$
\nabla \times \nabla \times \overline{\mathbf{G}}(\mathbf{r}, \omega)-k_{0}^{2}(\omega) \overline{\mathbf{G}}(\mathbf{r}, \omega)=\overline{\mathbf{I}} \delta(\mathbf{r}) .
$$

In the above equation the scattering effects of the air-soil surface interface are ignored but could, in principle, be incorporated in the Green's Dyadic.

The Born approximation to the electric field is the leading term in the Liouville Neumann expansion of the solution of the above integral equation and results in the following expression 
for the scattered field component of the electric field vector $[7,16]$ :

$$
\mathbf{E}_{s c a t}(\mathbf{r}, \omega)=-k_{0}^{2}(\omega) \int d^{3} r^{\prime} \overline{\mathbf{G}}\left(\mathbf{r}-\mathbf{r}^{\prime}, \omega\right) \cdot \mathbf{E}_{i n c}\left(\mathbf{r}^{\prime}, \omega\right) O\left(\mathbf{r}^{\prime}, \omega\right) .
$$

It is evident that the Born approximation results in the replacement of the total field $\mathbf{E}\left(\mathbf{r}^{\prime}, \omega\right)$ within the $\mathbf{r}^{\prime}$ integration by the incident field $\mathbf{E}_{i n c}\left(\mathbf{r}^{\prime}, \omega\right)$. Thus the Born approximation is valid for applications where multiple scattering effects can be neglected. The Born approximation is convenient for us because it allows a linear relation between $O(\mathbf{r}, \omega)$ and $\mathbf{E}_{\text {scat }}(\mathbf{r}, \omega)$, which will generally be easier to invert than the non-linear Eq.(2), where $\mathbf{E}_{\text {scat }}(\mathbf{r}, \omega)$ appears implicitly both on the left hand side and within the $\mathbf{r}^{\prime}$ integration. It has been shown [24] that DT using the Born approximation is adequate for quantitatively reconstructing objects with roughly $\left(0.8 \leq\left|\frac{\epsilon(\mathbf{r}, \omega)}{\epsilon_{0}(\omega)}\right| \leq 1.2\right)$, or 20 percent contrast, when the object size is on the order of a wavelength. This size to wavelength ratio is not unrealistic for GPR imaging, therefore the Born approximation should not be overly restrictive. Generally, as the object size decreases the allowable contrast increases. Thus our algorithm is suitable not only for imaging larger weak scatterers, but also for imaging smaller strong scatterers such as metal pipes. The Born approximation is often used, either implicitly or explicitly, for inverse problems in geophysics $[7,20,26,27]$ and it is assumed to be valid throughout this discussion.

To incorporate the characteristics of the transmitting and receiving antennas into the scattering equation, we first transform Eq. (4) into the spatial frequency domain, and then use Kerns' antenna scattering matrix formulation [13] to model the near-field interactions between antennas and scatterers. To convert Eq. (4) to the spatial frequency domain we employ the plane wave expansion for the Green's Dyadic [3]

$$
\overline{\mathbf{G}}\left(\mathbf{r}-\mathbf{r}^{\prime}, \omega\right)=\frac{i}{8 \pi^{2}} \int_{-\infty}^{\infty} \frac{d^{2} K}{\gamma(\mathbf{K}, \omega)}\left[\overline{\mathrm{I}}+\frac{\mathbf{k}^{+}(\omega) \mathbf{k}^{+}(\omega)}{k_{0}^{2}(\omega)}\right] e^{i \mathbf{k}^{+}(\omega) \cdot\left(\mathbf{r}-\mathbf{r}^{\prime}\right)},
$$

where we have assumed $z>z^{\prime}$ and where $\mathbf{K}=K_{x} \hat{\mathbf{x}}+K_{y} \hat{\mathbf{y}}$ is the spatial frequency variable, $\mathbf{k}^{+}(\omega)=\mathbf{K}+\gamma(\mathbf{K}, \omega) \hat{\mathbf{z}}$ is the wave vector for each plane wave $e^{i \mathbf{k}^{+}(\omega) \cdot\left(\mathbf{r}-\mathbf{r}^{\prime}\right)}$ in the expansion, and $\gamma(\mathbf{K}, \omega)= \pm \sqrt{k_{0}^{2}(\omega)-\mathbf{K} \cdot \mathbf{K}}$ with the sign chosen to render $\Im(\gamma) \geq 0$. For the lossless case (real $k_{0}$ ) the above plane wave expansion includes both homogeneous (propagating) plane waves $\left(|\mathbf{K}| \leq k_{0}\right)$ having purely real wave vectors $\mathbf{k}^{+}(\omega)$ and evanescent (non-propagating) plane waves $\left(|\mathbf{K}|>k_{0}\right)$ whose wave vectors are complex and which decay exponentially with increasing $\left(z-z^{\prime}\right)$. In the lossy case treated here (complex $k_{0}$ ) all plane waves will have complex wave vectors, however those corresponding to the higher spatial frequency range $|\mathbf{K}|>\Re\left[k_{0}\right]$ will decay most quickly with increasing $\left(z-z^{\prime}\right)$. Thus, in the lossy case the term "evanescent" is often applied to plane waves corresponding to the range $|\mathbf{K}|>\Re\left[k_{0}\right]$. In most formulations of DT the evanescent waves are discarded [7] since it is tacitly assumed that the measurements are conducted more than several wavelengths from the scatterers, i.e. $\left(z-z^{\prime}\right)>>\frac{2 \pi}{\Re\left[k_{0}\right]}$. In GPR imaging we should not discard evanescent components since 
objects of interest may be near the surface and wavelengths are relatively long (for adequate penetration), and therefore evanescent waves may contain valuable information [22].

We substitute Eq.(5) into Eq.(4) and evaluate the resulting expression at $z=0$ to find the scattered electric field at the ground surface. We then convert this expression to the spatial frequency domain by Fourier transforming relative to the $\mathbf{X}=x \hat{\mathbf{x}}+y \hat{\mathbf{y}}$ coordinate to obtain

$$
\begin{aligned}
\tilde{\mathbf{E}}_{\text {scat }}(\mathbf{K}, \omega) & =\frac{1}{(2 \pi)^{2}} \int_{-\infty}^{\infty} d^{2} X e^{-i \mathbf{K} \cdot \mathbf{x}}\left[\mathbf{E}_{s c a t}(\mathbf{r}, \omega)\right]_{z=0} \\
& =\frac{-i k_{0}^{2}(\omega)}{8 \pi^{2} \gamma(\mathbf{K}, \omega)} \int_{z^{\prime}<0} d^{3} r^{\prime} e^{-i \mathbf{k}^{+}(\omega) \cdot \mathbf{r}^{\prime}}\left[\overline{\mathbf{I}}+\frac{\mathbf{k}^{+}(\omega) \mathbf{k}^{+}(\omega)}{k_{0}^{2}(\omega)}\right] \cdot \mathbf{E}_{i n c}\left(\mathbf{r}^{\prime}, \omega\right) O\left(\mathbf{r}^{\prime}, \omega\right) .
\end{aligned}
$$

Using Kerns' scattering matrix formulation, a transmitting antenna centered at a position $\mathbf{X}_{\mathbf{j}} \equiv x_{j} \hat{\mathbf{x}}+y_{j} \hat{\mathbf{y}}$ on the ground surface and driven by matched terminal voltage $C(\omega)$ will give rise to the following plane wave expansion for the incident electric field propagating in the negative $\hat{\mathbf{z}}$ direction: $[13,23]$

$$
\mathbf{E}_{i n c}(\mathbf{r}, \omega)=C(\omega) \int_{-\infty}^{\infty} d^{2} K_{0} e^{-i \mathbf{K}_{\mathbf{0}} \cdot \mathbf{X}_{\mathbf{j}}} \mathbf{S}_{10}\left(\mathbf{K}_{\mathbf{0}}, \omega\right) e^{i \mathbf{k}_{\mathbf{0}}^{-}(\omega) \cdot \mathbf{r}}
$$

where $\mathbf{K}_{0}=K_{0 x} \hat{\mathbf{x}}+K_{0 y} \hat{\mathbf{y}}$ and $\mathbf{k}_{\mathbf{0}}^{-}(\omega)=\mathbf{K}_{\mathbf{0}}-\gamma\left(\mathbf{K}_{\mathbf{0}}, \omega\right) \hat{\mathbf{z}}$. Similarly, the matched terminal voltage at a receiver centered at $\mathbf{X}_{\mathbf{j}}$ (a monostatic experiment) is given by $[13,23]$

$$
V\left(\omega ; \mathbf{X}_{\mathbf{j}}\right)=\int_{-\infty}^{\infty} d^{2} K e^{i \mathbf{K} \cdot \mathbf{X}_{\mathbf{j}}} \mathbf{S}_{01}(\mathbf{K}, \omega) \cdot \tilde{\mathbf{E}}_{s c a t}(\mathbf{K}, \omega)
$$

In Eqs.(8) and (9), $\mathbf{S}_{10}$ and $\mathbf{S}_{01}$ are off-diagonal scattering matrix coefficients for the antennas, with each vector component corresponding to a Cartesian electric field polarization. It should be emphasized that Eqs.(8) and (9) are valid in the near field of the antennas, thus we need not make the point source approximation typical to other inversion algorithms of diffraction tomography. If the transmitting and receiving antennas are reciprocal, as would be the case for a typical monostatic radar system, then $[13,23]$

$$
Y_{0}(\omega) \mathbf{S}_{01}(\mathbf{K}, \omega)=\frac{\gamma(\mathbf{K}, \omega)}{\omega \mu_{0}} \mathbf{S}_{10}(-\mathbf{K}, \omega)
$$

where $Y_{0}$ is the antenna terminal admittance.

Combining Eqs.(6)-(10), the measured voltage $V(\omega)$ for a single monostatic experiment with the transmitter/receiver centered at $\mathbf{X}_{\mathbf{j}}$ is:

$$
\begin{aligned}
V\left(\omega ; \mathbf{X}_{\mathbf{j}}\right)= & P(\omega) \int_{-\infty}^{\infty} d^{2} K \int_{-\infty}^{\infty} d^{2} K_{0} e^{-i\left(\mathbf{K}_{\mathbf{0}}-\mathbf{K}\right) \cdot \mathbf{X}_{\mathbf{j}}} B\left(\mathbf{K}, \mathbf{K}_{\mathbf{0}} ; \omega\right) \\
& \cdot \int_{-\infty}^{0} d z^{\prime} e^{-i\left[\gamma(\mathbf{K}, \omega)+\gamma\left(\mathbf{K}_{0}, \omega\right)\right] z^{\prime}} \int_{-\infty}^{\infty} d^{2} X^{\prime} e^{-i\left(\mathbf{K}-\mathbf{K}_{\mathbf{0}}\right) \cdot \mathbf{X}^{\prime}} O\left(\mathbf{r}^{\prime}\right)
\end{aligned}
$$


where

$$
B\left(\mathbf{K}, \mathbf{K}_{0} ; \omega\right) \equiv \mathbf{S}_{10}(-\mathbf{K}, \omega) \cdot\left[\overline{\mathbf{I}}+\frac{\mathbf{k}^{+}(\omega) \mathbf{k}^{+}(\omega)}{k_{0}^{2}(\omega)}\right] \cdot \mathbf{S}_{10}\left(\mathbf{K}_{0}, \omega\right)
$$

and

$$
P(\omega)=\frac{-i C(\omega) k_{0}^{2}(\omega)}{8 \pi^{2} \omega Y_{0}(\omega) \mu_{0}}
$$

If the receiver in each experiment is spatially offset from the transmitter by a constant amount $\mathbf{X}_{\mathbf{0}}$, then we must multiply $B\left(\mathbf{K}, \mathbf{K}_{\mathbf{0}} ; \omega\right)$ given above by the factor $e^{i \mathbf{K} \cdot \mathbf{X}_{\mathbf{0}}}$.

Notice in Eq.(11) that the object function is expressed as a frequency independent (dispersion less) quantity $O(\mathbf{r}, \omega)=O(\mathbf{r})$. This assumption, although not necessary, allows us to couple the measured data at each frequency, thus incorporating more information into the mathematical inversions and leading to "better" solutions for the object function. There are alternatives to this approach that will work using the methodology of this report, including: (i) solve for the frequency dependent object function $O(\mathbf{r}, \omega)$ independently at each single radar frequency; (ii) treat the object function as the product of a known frequency dependent factor $\Theta(\omega)$ and an unknown frequency independent factor $O(\mathbf{r})$, then incorporate the known function $\Theta(\omega)$ into $P(\omega)$ given above, subsequently solving for the unknown $O(\mathbf{r})$ in the standard manner discussed below. In any case, we note that the assumption of a dispersion less object function in GPR is common (e.g. [27]) and models many important applications over relatively large frequency bands.

A typical GPR survey consists of multiple experiments, each corresponding to a different position $\mathbf{X}_{\mathbf{j}}$ for the transmitting/receiving antennas, thus we have a coupled set of equations, each in the form of Eq.(11). The objective of the present work is to estimate the object function $O(\mathbf{r})$ by inverting the coupled equations using the regularized pseudo inverse formula as described below. 


\section{Data inversion for 3D surveys.}

In this section we construct a mathematical operator formalism for the coupled set of Eqs.(11), where each equation corresponds to a different transmitter/receiver position. This mathematical framework allows us to use well known linear inversion methods to analytically solve for the object function in terms of the measured data.

We consider a three dimensional survey where a series of monostatic experiments are performed over the ground surface at evenly spaced locations corresponding to points on a two dimensional grid. For each experiment we store data from $N$ different excitation frequencies $\omega_{n}$. If the grid spacing is small enough to satisfy the Nyquist sampling criterion for the voltage measurements, we can treat the transmitter/receiver position as a continuous variable $\mathbf{X}=(x, y)$ (below we discuss the spatial sampling requirements in further detail). Thus, from Eq.(11) the measured voltage as a function of frequency and transmitter/receiver position is

$$
\begin{aligned}
V\left(\omega_{n} ; \mathbf{X}\right)= & P\left(\omega_{n}\right) \int_{-\infty}^{\infty} d^{2} K \int_{-\infty}^{\infty} d^{2} K_{0} e^{-i\left(\mathbf{K}_{0}-\mathbf{K}\right) \cdot \mathbf{x}} B\left(\mathbf{K}, \mathbf{K}_{0} ; \omega_{n}\right) \\
& \cdot \int_{-\infty}^{0} d z^{\prime} e^{-i\left[\gamma\left(\mathbf{K}, \omega_{n}\right)+\gamma\left(\mathbf{K}_{0}, \omega_{n}\right)\right] z^{\prime}} \int_{-\infty}^{\infty} d^{2} X^{\prime} e^{-i\left(\mathbf{K}-\mathbf{K}_{0}\right) \cdot \mathbf{X}^{\prime}} O\left(\mathbf{r}^{\prime}\right) .
\end{aligned}
$$

We make the change of variable $\overline{\mathbf{K}}=\mathbf{K}-\mathbf{K}_{\mathbf{0}}$, and drop the bar notation on $\overline{\mathbf{K}}$ to yield

$$
\begin{aligned}
V\left(\omega_{n} ; \mathbf{X}\right)= & \int_{-\infty}^{\infty} d^{2} K e^{i \mathbf{K} \cdot \mathbf{x}} P\left(\omega_{n}\right) \int_{-\infty}^{\infty} d^{2} K_{0} B\left(\mathbf{K}+\mathbf{K}_{\mathbf{0}}, \mathbf{K}_{\mathbf{0}} ; \omega_{n}\right) \\
& \cdot \int_{-\infty}^{0} d z^{\prime} e^{-i\left[\gamma\left(\mathbf{K}+\mathbf{K}_{\mathbf{0}}, \omega_{n}\right)+\gamma\left(\mathbf{K}_{\mathbf{0}}, \omega_{n}\right)\right] z^{\prime}} \int_{-\infty}^{\infty} d^{2} X^{\prime} e^{-i \mathbf{K} \cdot \mathbf{X}^{\prime}} O\left(\mathbf{r}^{\prime}\right) .
\end{aligned}
$$

We now spatially Fourier transform Eq.(17) with respect to the $\mathbf{X}$ variable to obtain

$$
\begin{aligned}
\tilde{V}\left(\omega_{n} ; \mathbf{K}\right) & \equiv \frac{1}{(2 \pi)^{2}} \int_{-\infty}^{\infty} d^{2} X e^{-i \mathbf{K} \cdot \mathbf{x}_{V}}\left(\omega_{n} ; \mathbf{X}\right) \\
& =P\left(\omega_{n}\right) \int_{-\infty}^{\infty} d^{2} K_{0} B\left(\mathbf{K}+\mathbf{K}_{\mathbf{0}}, \mathbf{K}_{\mathbf{0}} ; \omega_{n}\right) \\
& \cdot \int_{-\infty}^{0} d z^{\prime} e^{-i\left[\gamma\left(\mathbf{K}+\mathbf{K}_{0}, \omega_{n}\right)+\gamma\left(\mathbf{K}_{0}, \omega_{n}\right)\right] z^{\prime}} \int_{-\infty}^{\infty} d^{2} X^{\prime} e^{-i \mathbf{K} \cdot \mathbf{X}^{\prime}} O\left(\mathbf{r}^{\prime}\right) .
\end{aligned}
$$

It is important to define Hilbert spaces for the various quantities entering into Eq.(19) and to express this equation in compact operator formalism. Thus, we introduce the vector space $U$ of object functions $O(\mathbf{r})$ and the vector space $Y$ of transformed measured voltages $\tilde{V}\left(\omega_{n} ; \mathbf{K}\right)$. We will employ the standard $L^{2}$ inner products in both spaces and will assume the elements of each space to have finite $L^{2}$ norms, i.e.

- $O(\mathbf{r}) \in U$, where $U$ is the space of square integrable functions on $-\infty<(x, y)<\infty$, $-\infty<z<0$ 
- $\tilde{V}\left(\omega_{n} ; \mathbf{K}\right) \in Y$, where $Y$ is the direct product space of square integrable functions on $-\infty<\left(K_{x}, K_{y}\right)<\infty$ with the finite dimensional vector space $Y_{0}$ of functions of the discrete variable $\omega_{n}$.

With these definitions we can write Eq.(19) in the compact form

$$
\tilde{V}\left(\omega_{n} ; \mathbf{K}\right)=H O\left(\omega_{n} ; \mathbf{K}\right),
$$

where $H$ is a linear operator which maps $U$ into $Y$.

Our goal is to find the object function $O\left(\mathrm{r}^{\prime}\right)$ which satisfies the set of Eqs.(22). Since we use only a finite number of excitation frequencies, the set of equations (22) are under determined so that there are an infinite number of object functions which will produce the data. The minimum $L^{2}$ norm solution is approximately found by applying the regularized pseudo inverse operator $[1,2,12,17]$ to the data, i.e.

$$
\hat{O}_{\beta}(\mathbf{r})=H^{\dagger}\left[H H^{\dagger}+\beta I\right]^{-1} \tilde{V}(\mathbf{r})
$$

where $H^{\dagger}$ is the Hermitian adjoint of $H$. In the limit as $\beta$ goes to zero, $\hat{O}_{\beta}(\mathbf{r})$ is equal to the minimum $L^{2}$ norm solution to Eq. $(22)^{1}$. The number $\beta$ is known as the TikhonovPhillips regularization parameter $[21,17]$, and is necessary to stabilize the inversion against noise from measurements or numerical truncations. The regularization parameter is also necessary in the event that $\left(H H^{\dagger}\right)^{-1}$ does not exist. Generally there is a tradeoff between selecting $\beta$ small enough such that $\hat{O}_{\beta}(\mathbf{r})$ approximately satisfies the data yet large enough that the inversion is stable, and typically $\beta$ is selected by trial and error. We show below that Eq. (23) is in the form of a filtered backpropagation operation, where $\left[H H^{\dagger}+\beta I\right]^{-1}$ is the filtering operator and the $H^{\dagger}$ is a coherent sum over frequencies of the filtered, back propagated, data.

Before proceeding with the inversion algorithm, we should make several comments about our solution strategy. First, in GPR imaging there will be a significant amount of noise associated both with the receiver electronics and scattering from ground clutter, and this noise may profoundly affect computed solutions. One can modify Eq.(22) to include this additive noise component $n$ explicitly as follows:

$$
\tilde{V}=H O+n \text {. }
$$

\footnotetext{
${ }^{1}$ In practice one does not have access to an infinite number of monostatic measurements, rather the received voltage is sampled at discrete spatial intervals over a finite area of the $x y$ plane. Representing this sampled voltage as $\bar{V}=R F^{-1} \tilde{V}=R F^{-1} H O$, where $R$ is the sampling operator and $F^{-1}$ is the inverse Fourier transform, we can show that $\hat{O}_{\beta=0}$ from Eq.(23) is also the minimum $L^{2}$ norm solution to $\bar{V}=R F^{-1} H O$ if the linear operator $\left(R F^{-1}\right)$ can be inverted. In practice, $\left(R F^{-1}\right)$ can be inverted if the Nyquist sampling criterion is satisfied and the spatial extent of the sampling area is large relative to the support region dimensions (depth and horizontal) of the object function.
} 
The minimum variance (Wiener filtering) solution $[2,12]$ to this equation is

$$
\hat{O}_{W}=R_{V} H^{\dagger}\left[H R_{V} H^{\dagger}+R_{n}\right]^{-1} \tilde{V}
$$

where $\tilde{V}$ and $n$ are assumed to be zero-mean random processes having covariances $R_{V}$ and $R_{n}$, respectively. If $\tilde{V}$ and $n$ are Gaussian white processes with variances $\sigma_{V}^{2}$ and $\sigma_{n}^{2}$, respectively (the Gaussian white assumption may be sensible if no other information is available), then Eq.(25) reduces to $[2,12]$

$$
\hat{O}_{W}=H^{\dagger}\left[H H^{\dagger}+\sigma_{n}^{2} / \sigma_{V}^{2}\right]^{-1} \tilde{V} .
$$

Note that Eqs.(23) and (26) are identical for $\beta=\sigma_{n}^{2} / \sigma_{V}^{2}$, and thus we expect that the optimum value for $\beta$ in Tikhonov-Phillips regularization is roughly proportional to the noise variance. Note that there are other robust alternatives to Tikhonov-Phillips regularization and Wiener filtering, for example the reduced rank pseudo inverse operator $[1,10]$. We choose the method of Tikhonov-Phillips regularization because it allows us to derive analytical formulas.

Let us now analytically expand the operator notation in Eq.(23) to obtain explicitly the pseudo inverse solution. Given the vector space definitions, the Hermitian adjoint $H^{\dagger}$ of $H$ maps the space $Y$ onto the space $U$ so that

$$
<H O_{\beta}, \tilde{V}>_{Y}=<O_{\beta}, H^{\dagger} \tilde{V}>_{U} .
$$

From this inner product relation the action of $H^{\dagger}$ is found to be

$$
\begin{aligned}
H^{\dagger} \hat{\tilde{V}}\left(\mathbf{r}^{\prime}\right) & \equiv \hat{O}_{\beta}\left(\mathbf{r}^{\prime}\right) \\
& =\sum_{n=1}^{N} P^{*}\left(\omega_{n}\right) \int_{-\infty}^{\infty} d^{2} K^{\prime} \int_{-\infty}^{\infty} d^{2} K_{0}^{\prime} B^{*}\left(\mathbf{K}^{\prime}+\mathbf{K}_{0}^{\prime}, \mathbf{K}_{0}^{\prime} ; \omega_{n}\right) \\
& \cdot e^{i\left[\gamma^{*}\left(\mathbf{K}^{\prime}+\mathbf{K}_{\mathbf{0}}^{\prime}, \omega_{n}\right)+\gamma^{*}\left(\mathbf{K}_{\mathbf{0}}^{\prime}, \omega_{n}\right)\right] z^{\prime}} e^{i \mathbf{K}^{\prime} \cdot \mathbf{X}^{\prime}} \hat{\tilde{V}}\left(\omega_{n} ; \mathbf{K}^{\prime}\right)
\end{aligned}
$$

where the $(*)$ denotes the complex conjugate, $\mathbf{r}^{\prime}=\left(\mathbf{X}^{\prime}, z^{\prime}\right)$, and where we have added a "hat" to $\tilde{V}$ for later notational purposes. In typical filtered backpropagation algorithms $[4,6,7]$ the backpropagation operation is the adjoint of the forward propagation. Similarly, each term in the summation in Eq.(28) is the adjoint of the forward scattering operator at a single excitation frequency. Because of this analogy, we refer to each term in the summation as a backpropagation, although this is not true in the usual sense.

Let us now consider the operation $\hat{\tilde{V}}=\left[H H^{\dagger}+\beta I\right]^{-1} \tilde{V}$, which corresponds to the filtering operation in standard DT filtered backpropagation algorithms, where $\hat{\tilde{V}}$ is the filtered data and $\tilde{V}$ is the raw, un filtered, data. From Eqs. (19) and (28), the inverse of the filtering 
operation is

$$
\begin{aligned}
\tilde{V}\left(\omega_{m} ; \mathbf{K}\right)= & {\left[H H^{\dagger}+\beta I\right] \hat{\tilde{V}}\left(\omega_{m} ; \mathbf{K}\right) } \\
= & \sum_{n=1}^{N} P\left(\omega_{m}\right) P^{*}\left(\omega_{n}\right) \int_{-\infty}^{\infty} d^{2} K^{\prime} \hat{\tilde{V}}\left(\omega_{n} ; \mathbf{K}^{\prime}\right) \\
& \cdot \int_{-\infty}^{\infty} d^{2} K_{0} \int_{-\infty}^{\infty} d^{2} K_{0}^{\prime} B\left(\mathbf{K}+\mathbf{K}_{\mathbf{0}}, \mathbf{K}_{\mathbf{0}} ; \omega_{m}\right) B^{*}\left(\mathbf{K}^{\prime}+\mathbf{K}_{0}^{\prime}, \mathbf{K}_{\mathbf{0}}^{\prime} ; \omega_{n}\right) \\
& \cdot \int_{-\infty}^{0} d z^{\prime} e^{-i\left[\gamma\left(\mathbf{K}+\mathbf{K}_{\mathbf{0}}, \omega_{m}\right)+\gamma\left(\mathbf{K}_{\mathbf{0}}, \omega_{m}\right)-\gamma^{*}\left(\mathbf{K}^{\prime}+\mathbf{K}_{\mathbf{0}}^{\prime}, \omega_{n}\right)-\gamma^{*}\left(\mathbf{K}_{\mathbf{0}}^{\prime}, \omega_{n}\right)\right] z^{\prime}} \\
& \cdot \int_{-\infty}^{\infty} d^{2} X^{\prime} e^{-i\left(\mathbf{K}-\mathbf{K}^{\prime}\right) \cdot \mathbf{X}^{\prime}} \\
+ & \beta \hat{\tilde{V}}\left(\omega_{m} ; \mathbf{K}\right) .
\end{aligned}
$$

The integral over $\mathrm{X}^{\prime}$ reduces to a Dirac delta function, and the integral over $z^{\prime}$ can be solved since $\Im(\gamma)>0$, therefore

$$
\tilde{V}\left(\omega_{m} ; \mathbf{K}\right)=\sum_{n=1}^{N} R_{m n}(\mathbf{K}) \hat{\tilde{V}}\left(\omega_{n} ; \mathbf{K}\right)+\beta \hat{\tilde{V}}\left(\omega_{m} ; \mathbf{K}\right)
$$

where

$$
R_{m n}(\mathbf{K})=\int_{-\infty}^{\infty} d^{2} K_{0} \int_{-\infty}^{\infty} d^{2} K_{0}^{\prime} \frac{4 i \pi^{2} P\left(\omega_{m}\right) P^{*}\left(\omega_{n}\right) B\left(\mathbf{K}+\mathbf{K}_{\mathbf{0}}, \mathbf{K}_{\mathbf{0}} ; \omega_{m}\right) B^{*}\left(\mathbf{K}+\mathbf{K}_{0}^{\prime}, \mathbf{K}_{\mathbf{0}}^{\prime} ; \omega_{n}\right)}{\left[\gamma\left(\mathbf{K}+\mathbf{K}_{\mathbf{0}}, \omega_{m}\right)+\gamma\left(\mathbf{K}_{\mathbf{0}}, \omega_{m}\right)-\gamma^{*}\left(\mathbf{K}+\mathbf{K}_{0}^{\prime}, \omega_{n}\right)-\gamma^{*}\left(\mathbf{K}_{\mathbf{0}}^{\prime}, \omega_{n}\right)\right]}
$$

For each value of $\mathbf{K}$ this set of equations can be written in the matrix notation

$$
\left\{\begin{array}{c}
\tilde{V}\left(\omega_{1} ; \mathbf{K}\right) \\
\tilde{V}\left(\omega_{2} ; \mathbf{K}\right) \\
\vdots \\
\tilde{V}\left(\omega_{N} ; \mathbf{K}\right)
\end{array}\right\}=\left\{\begin{array}{cccc}
R_{11}(\mathbf{K})+\beta & R_{12}(\mathbf{K}) & \cdots & R_{1 N}(\mathbf{K}) \\
R_{21}(\mathbf{K}) & R_{22}(\mathbf{K})+\beta & \cdots & R_{2 N}(\mathbf{K}) \\
\vdots & \vdots & \ddots & \vdots \\
R_{N 1}(\mathbf{K}) & R_{N 2}(\mathbf{K}) & \cdots & R_{N N}(\mathbf{K})+\beta
\end{array}\right\}\left\{\begin{array}{c}
\hat{\tilde{V}}\left(\omega_{1} ; \mathbf{K}\right) \\
\hat{\tilde{V}}\left(\omega_{2} ; \mathbf{K}\right) \\
\vdots \\
\hat{\tilde{V}}\left(\omega_{N} ; \mathbf{K}\right)
\end{array}\right\}
$$

which can be inverted by standard methods of linear algebra to yield the filtered data $\hat{\tilde{V}}$ in terms of the raw, un filtered data $\tilde{V}$. The regularized pseudo inverse solution $\hat{O}_{\beta}(\mathbf{r})$ can then be found by operating on the filtered data by the backpropagation operator $H^{\dagger}$ as given in Eq. (28).

As an example of the object function reconstruction, let us consider the case where there are two radar frequencies $\omega_{1}$ and $\omega_{2}$. Inverting the $2 \times 2$ version of Eq.(38), we have the following solutions for the filtered data:

$$
\hat{\tilde{V}}\left(\omega_{1} ; \mathbf{K}\right)=\frac{\left(R_{22}(\mathbf{K})+\beta\right)}{\Delta(\mathbf{K})} \tilde{V}\left(\omega_{1} ; \mathbf{K}\right)-\frac{R_{12}(\mathbf{K})}{\Delta(\mathbf{K})} \tilde{V}\left(\omega_{2} ; \mathbf{K}\right)
$$


and

$$
\hat{\tilde{V}}\left(\omega_{2} ; \mathbf{K}\right)=-\frac{R_{21}(\mathbf{K})}{\Delta(\mathbf{K})} \tilde{V}\left(\omega_{1} ; \mathbf{K}\right)+\frac{\left(R_{11}(\mathbf{K})+\beta\right)}{\Delta(\mathbf{K})} \tilde{V}\left(\omega_{2} ; \mathbf{K}\right)
$$

where

$$
\Delta(\mathbf{K})=\left[R_{11}(\mathbf{K})+\beta\right]\left[R_{22}(\mathbf{K})+\beta\right]-R_{21}(\mathbf{K}) R_{12}(\mathbf{K}) .
$$

According to Eq.(23), we then reconstruct the object function $\hat{O}_{\beta}(\mathbf{r})$ by back propagating the filtered data using Eq.(28) with $N=2$. 


\section{Data inversion for 2.5D surveys.}

The 2.5 dimensional case corresponds to assuming a two dimensional object function $O(x, z)$, invariant in the $\hat{\mathbf{y}}$ direction, but fully three dimensional incident and scattered wave fields. We can treat the 2.5 dimensional case in a manner completely analogous to the three dimensional analysis of Section 3. Whereas for the three dimensional case we conducted a series of monostatic experiments over a two dimensional grid on the $x y$ plane, here we conduct experiments at intervals along a one dimensional line corresponding to the $\hat{\mathbf{x}}$ axis. From the scattered field measurements we wish to reconstruct the object function $O(x, z)$. The receiver voltage for each experiment is related to the object function by Eq.(11). We emphasize that the antennas are three dimensional, such that the incident and scattered electric fields vary in three dimensions and have three Cartesian polarizations.

For each experiment we store data from $N$ different excitation frequencies $\omega_{n}$. We assume the interval spacing along the $\hat{\mathrm{x}}$ axis is small enough to satisfy the Nyquist sampling criterion for the voltage measurements, thus we can treat the measured voltage $V\left(\omega_{n} ; x\right)$ as a function of the continuous variable $x$ (the discussion of the sampling requirements from the footnote in Section 3 is valid here also). The data is defined as the spatial Fourier transform relative to the $x$ coordinate of the measured voltage. By methods similar to the three dimensional analysis, this data is found to be

$$
\begin{aligned}
\tilde{V}\left(\omega_{n} ; K_{x}\right) & \equiv \frac{1}{2 \pi} \int_{-\infty}^{\infty} d x e^{-i K_{x} x} V\left(\omega_{n} ; x\right) \\
& =2 \pi P\left(\omega_{n}\right) \int_{-\infty}^{\infty} d^{2} K_{0} B\left(K_{x} \hat{\mathbf{x}}+\mathbf{K}_{0}, \mathbf{K}_{\mathbf{0}} ; \omega_{n}\right) \\
& \cdot \int_{-\infty}^{0} d z^{\prime} e^{-i\left[\gamma\left(K_{x} \hat{\mathbf{x}}+\mathbf{K}_{0}, \omega_{n}\right)+\gamma\left(\mathbf{K}_{0}, \omega_{n}\right)\right) z^{\prime}} \int_{-\infty}^{\infty} d x^{\prime} e^{-i K_{x} x^{\prime}} O\left(x^{\prime}, z^{\prime}\right) \\
& =A O\left(\omega_{n} ; K_{x}\right) .
\end{aligned}
$$

Here we use the Hilbert space definitions

- $O(x, z) \in W$, where $W$ is the space of square integrable functions on $-\infty<x<\infty$, $-\infty<z<0$

- $\tilde{V}\left(\omega_{n} ; K_{x}\right) \in Z$, where $Z$ is the direct product space of square integrable functions on $-\infty<K_{x}<\infty$ with the finite dimensional vector space $Y_{0}$ of functions of the discrete variable $\omega_{n}$

- $A$ is a linear operator which maps $W$ into $Z$.

We seek the regularized pseudo inverse solution for the object function

$$
\begin{aligned}
\hat{O}_{\beta}(x, z) & =A^{\dagger}\left[A A^{\dagger}+\beta I\right]^{-1} \tilde{V}(x, z) \\
& =A^{\dagger} \hat{\tilde{V}}(x, z) .
\end{aligned}
$$


Given the vector space definitions, the adjoint of $A$ is specified by the action

$$
\begin{aligned}
A^{\dagger} \hat{\tilde{V}}\left(x^{\prime}, z^{\prime}\right) & \equiv \hat{O}_{\beta}\left(x^{\prime}, z^{\prime}\right) \\
& =\sum_{n=1}^{N} 2 \pi P^{*}\left(\omega_{n}\right) \int_{-\infty}^{\infty} d K_{x}^{\prime} \int_{-\infty}^{\infty} d^{2} K_{0}^{\prime} B^{*}\left(K_{x}^{\prime} \hat{\mathbf{x}}+\mathbf{K}_{0}^{\prime}, \mathbf{K}_{0}^{\prime} ; \omega_{n}\right) \\
& \cdot e^{i\left[\gamma^{*}\left(K_{x}^{\prime} \hat{\mathbf{x}}+\mathbf{K}_{0}^{\prime}, \omega_{n}\right)+\gamma^{*}\left(\mathbf{K}_{0}^{\prime}, \omega_{n}\right)\right] z^{\prime}} e^{i K_{x}^{\prime} x^{\prime}} \hat{\tilde{V}}\left(\omega_{n} ; K_{x}^{\prime}\right) .
\end{aligned}
$$

The filtered data $\hat{\tilde{V}}$ and the raw, un filtered, data $\tilde{V}$ are related by the filtering relation $\hat{\tilde{V}}=\left[A A^{\dagger}+\beta I\right]^{-1} \tilde{V}$, which is expanded by combining Eqs. (42) and (48) and evaluating the $x^{\prime}$ and $z^{\prime}$ integrals as in Section 3. In this manner we obtain the following relation between the filtered and un filtered data:

$$
\tilde{V}\left(\omega_{m} ; K_{x}\right)=\sum_{n=1}^{N} Q_{m n}\left(K_{x}\right) \hat{\tilde{V}}\left(\omega_{n} ; K_{x}\right)+\beta \hat{\tilde{V}}\left(\omega_{m} ; K_{x}\right)
$$

where

$Q_{m n}\left(K_{x}\right)=\int_{-\infty}^{\infty} d^{2} K_{0} \int_{-\infty}^{\infty} d^{2} K_{0}^{\prime} \frac{8 i \pi^{3} P\left(\omega_{m}\right) P^{*}\left(\omega_{n}\right) B\left(K_{x} \hat{\mathbf{x}}+\mathbf{K}_{0}, \mathbf{K}_{0} ; \omega_{m}\right) B^{*}\left(K_{x} \hat{\mathbf{x}}+\mathbf{K}_{0}^{\prime}, \mathbf{K}_{0}^{\prime} ; \omega_{n}\right)}{\left[\gamma\left(K_{x} \hat{\mathbf{x}}+\mathbf{K}_{0}, \omega_{m}\right)+\gamma\left(\mathbf{K}_{0}, \omega_{m}\right)-\gamma^{*}\left(K_{x} \hat{\mathbf{x}}+\mathbf{K}_{0}^{\prime}, \omega_{n}\right)-\gamma^{*}\left(\mathbf{K}_{0}^{\prime}, \omega_{n}\right)\right]}$

For each value of $K_{x}$, Eq.(51) can be written in the matrix notation

$$
\left\{\begin{array}{c}
\tilde{V}\left(\omega_{1} ; K_{x}\right) \\
\tilde{V}\left(\omega_{2} ; K_{x}\right) \\
\vdots \\
\tilde{V}\left(\omega_{N} ; K_{x}\right)
\end{array}\right\}=\left\{\begin{array}{cccc}
Q_{11}\left(K_{x}\right)+\beta & Q_{12}\left(K_{x}\right) & \cdots & Q_{1 N}\left(K_{x}\right) \\
Q_{21}\left(K_{x}\right) & Q_{22}\left(K_{x}\right)+\beta & \cdots & Q_{2 N}\left(K_{x}\right) \\
\vdots & \vdots & \ddots & \vdots \\
Q_{N 1}\left(K_{x}\right) & Q_{N 2}\left(K_{x}\right) & \cdots & Q_{N N}\left(K_{x}\right)+\beta
\end{array}\right\}\left\{\begin{array}{c}
\hat{\tilde{V}}\left(\omega_{1} ; K_{x}\right) \\
\hat{\tilde{V}}\left(\omega_{2} ; K_{x}\right) \\
\vdots \\
\tilde{V}\left(\omega_{N} ; K_{x}\right)
\end{array}\right\}
$$

which can be inverted by standard methods of linear algebra to yield the filtered data $\hat{\tilde{V}}$ in terms of the raw, un filtered data $\tilde{V}$. The regularized pseudo inverse solution $\hat{O}_{\beta}(\mathrm{r})$ can then be found by operating on the filtered data by the operator $A^{\dagger}$ as given in Eq.(48). 


\section{Computed results from synthetic data.}

We present here the results of computer simulations designed to test the reconstruction algorithm. For simplicity, we treat the two dimensional problem where the transmitted electric field has only a $\hat{\mathbf{y}}$ polarization, and the antennas and scatterers do not vary in the $\hat{\mathbf{y}}$ direction ${ }^{2}$. Thus, $\mathbf{K}=K \hat{\mathbf{x}}, \mathbf{K}_{0}=K_{0} \hat{\mathbf{x}}, \mathbf{X}=x \hat{\mathbf{x}}$, and $\mathbf{S}_{10}(\mathbf{K}, \omega)=S_{10}(K, \omega) \hat{\mathbf{y}}$. Using this two dimensional model, we can simplify all results in Sections 2 and 3 by converting vector quantities to scalars, reducing by one the dimensions of all integrations over $K, K_{0}$, and $\mathbf{r}$, and changing some constant factors.

More explicitly, the forward model for the two dimensional case can be shown to be

$$
\begin{aligned}
\tilde{V}\left(\omega_{n} ; K_{x}\right)= & 2 \pi P\left(\omega_{n}\right) \int_{-\infty}^{\infty} d K_{0} B\left(K_{x}+K_{0}, K_{0} ; \omega_{n}\right) \\
& \cdot \int_{-\infty}^{0} d z^{\prime} e^{-i\left[\gamma\left(K_{x}+K_{0}, \omega_{n}\right)+\gamma\left(K_{0}, \omega_{n}\right)\right] z^{\prime}} \int_{-\infty}^{\infty} d x^{\prime} e^{-i K_{x} x^{\prime}} O\left(x^{\prime}, z^{\prime}\right) .
\end{aligned}
$$

As we discussed in the previous sections, the object function is reconstructed by a filtered backpropagation algorithm. The filtering operation consists of inverting the matrix Eq.(53) for each value of $K_{x}$ where, for the two dimensional case, the matrix elements can be shown to be

$$
Q_{m n}\left(K_{x}\right)=\int_{-\infty}^{\infty} d K_{0} \int_{-\infty}^{\infty} d K_{0}^{\prime} \frac{8 i \pi^{3} P\left(\omega_{m}\right) P^{*}\left(\omega_{n}\right) B\left(K_{x}+K_{0}, K_{0} ; \omega_{m}\right) B^{*}\left(K_{x}+K_{0}^{\prime}, K_{0}^{\prime} ; \omega_{n}\right)}{\left[\gamma\left(K_{x}+K_{0}, \omega_{m}\right)+\gamma\left(K_{0}, \omega_{m}\right)-\gamma^{*}\left(K_{x}+K_{0}^{\prime}, \omega_{n}\right)-\gamma^{*}\left(K_{0}^{\prime}, \omega_{n}\right)\right]} .
$$

Once the data is filtered, the object function is reconstructed by the following two dimensional backpropagation, analogous to Eqs.(28) and (48):

$$
\begin{aligned}
\hat{O}_{\beta}\left(x^{\prime}, z^{\prime}\right)= & \sum_{n=1}^{N} 2 \pi P^{*}\left(\omega_{n}\right) \int_{-\infty}^{\infty} d K_{x}^{\prime} \int_{-\infty}^{\infty} d K_{0}^{\prime} B^{*}\left(K_{x}^{\prime}+K_{0}^{\prime}, K_{0}^{\prime} ; \omega_{n}\right) \\
& \cdot e^{i\left[\gamma^{*}\left(K_{x}^{\prime}+K_{0}^{\prime}, \omega_{n}\right)+\gamma^{*}\left(K_{0}^{\prime}, \omega_{n}\right)\right] z^{\prime}} e^{i K_{x}^{\prime} x^{\prime}} \hat{\tilde{V}}\left(\omega_{n} ; K_{x}^{\prime}\right) .
\end{aligned}
$$

Fig.2 shows a purely real two dimensional object function $O(x, z)$ which is embedded in a soil background having a penetration depth of 3 meters at all frequencies. The value of the object function is $O(x, z)=0.2$ within the cross region at all frequencies, therefore the dielectric contrast is $\frac{\epsilon(\mathbf{r}, \omega)}{\epsilon_{0}(\omega)}=0.8$. The antennas are characterized as being fairly directional, with (two dimensional scalar) transmitting coefficients given by ${ }^{3}$

$$
S_{10}(K, \omega)=e^{-a(\omega) K^{2}} / \gamma(K, \omega),
$$

where $a(\omega)$ is a constant chosen such that $e^{-a(\omega)\left[\Re\left(k_{0}\right)\right]^{2}} \approx 1 / 10$. For each simulation, the synthetic data is obtained by numerically applying Eq.(54) to the object of Fig.2.

\footnotetext{
${ }^{2}$ In practice, this model will be valid for one dimensional linear antennas, and scatterers that vary slowly in the $\hat{\mathbf{y}}$ direction.

${ }^{3}$ For comparison, it can be shown from the plane wave expansion of the Green's Dyadic in Eq.(5) that
} 


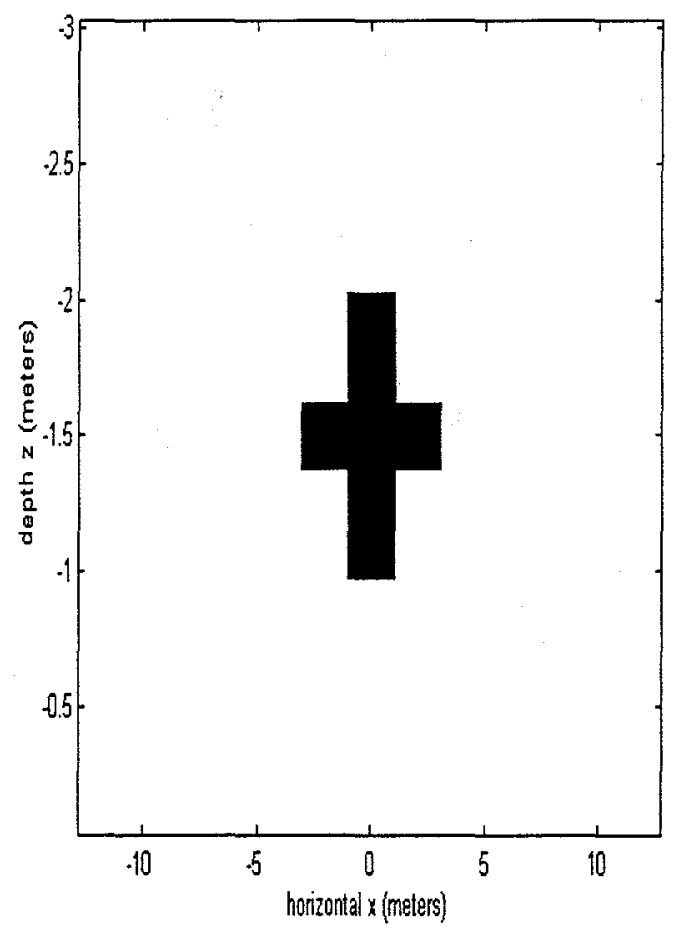

Figure 1: Original object function for frequency domain simulations. $O(x, z)=0.2$ within the cross region at all frequencies. 
We initially compute a reconstruction based on a single radar frequency corresponding to the wavelength $\lambda=1.5$ meters. To accomplish this task we first filter the data according to the equation

$$
\hat{\tilde{V}}\left(\omega_{1} ; K_{x}\right)=\frac{\tilde{V}\left(\omega_{1} ; K_{x}\right)}{Q_{11}\left(K_{x}\right)+\beta},
$$

(with $Q_{11}\left(K_{x}\right)$ given in Eq.(56)) which is the inverse of the $1 \times 1$ version of Eqs.(53). The minimum norm reconstruction is then obtained by backpropagation as defined by Eq.(57) with $N=1$. The magnitude of the filter $1 /\left(Q_{11}\left(K_{x}\right)+\beta\right)$ is plotted vs. the spatial frequency $K_{x}$ in Fig. 3 for several different values of the regularization parameter $\beta$. It is evident that this filter tends to amplify the high spatial frequency components of the data relative to the low frequency components. Without regularization, high frequency components from additive noise will be amplified without bound, thus leading to unstable inversions. By using a non-zero $\beta$ this amplification is limited, thus stabilizing the inversion. The real part of the minimum norm reconstruction is shown in Fig. 4, where $\beta=1 e-9$ was chosen by trial and error. The criterion used in the selection of $\beta$ was that both the original object of Fig. 2 and the reconstruction of Fig. 4 should yield nearly identical data since they are both solutions to Eq.(54). Note from Fig. 4 that the outline of the original object function is evident, however there is considerable periodic ambiguity in the image.

Next we compute a reconstruction based on 9 frequencies corresponding to $0.375 m<\lambda<$ $3.375 \mathrm{~m}$ using the $N=9$ versions of Eqs. (57) and (53). The regularization parameter is set to $\beta=1 e-9$, as before. It can be noted from the real part of the reconstruction, shown in Fig. 5 , that by using multiple frequencies the periodic ambiguity observed in Fig. 4 is diminished, and we obtain a fairly good representation of the original scattering object. In Fig. 6 we plot the cross section of Fig. 5 along the line $x=0$, as well as cross sections from reconstructions using 15 and 21 frequencies corresponding to the range $0.375 m<\lambda<3.375 \mathrm{~m}$. It is evident that increasing the number of frequencies leads to a significant quantitative improvement. This concept is supported also by Fig. 7, which shows decreasing error $=\int d^{2} r\left|O(\mathbf{r})-\hat{O}_{\beta}(\mathbf{r})\right|^{2}$ with increasing numbers of frequencies corresponding to the range $0.375 m<\lambda<3.375 m$.

As a test that the minimum norm reconstruction is consistent with the data, and therefore satisfies Eq.(54), we implement the forward scattering relation of Eq.(54) on both the original object function of Fig. 2 and the reconstructed object function of Fig. 5 computed from 9 frequencies. The real part of the data $\tilde{V}\left(\omega_{n} ; K_{x}\right)$ is plotted in Figs. 8a and 8b for both the original and reconstructed object functions, at 2 of the 9 frequencies corresponding to $\lambda=1.125 \mathrm{~m}$ and $\lambda=2.25 \mathrm{~m}$ (to avoid redundancy, data from all the frequencies is not plotted). Based on the excellent match obtained between data from the original and reconstructed objects at all frequencies (the plots are almost completely overlapping in the

a thin linear antenna, exciting an omni-directional field, will have the transmitting coefficient $S_{10}(K, \omega)=$ $1 / \gamma(K, \omega)$. The exponential numerator in Eq.(59) makes the antenna directional. 


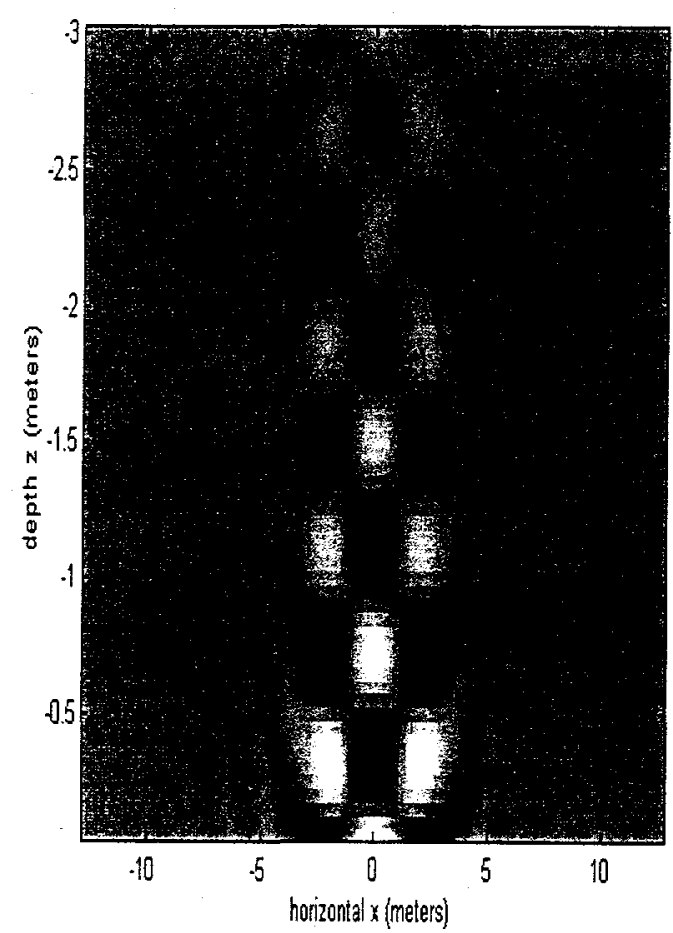

Figure 2: Original object function for frequency domain simulations. $O(x, z)=0.2$ within the cross region at all frequencies. 


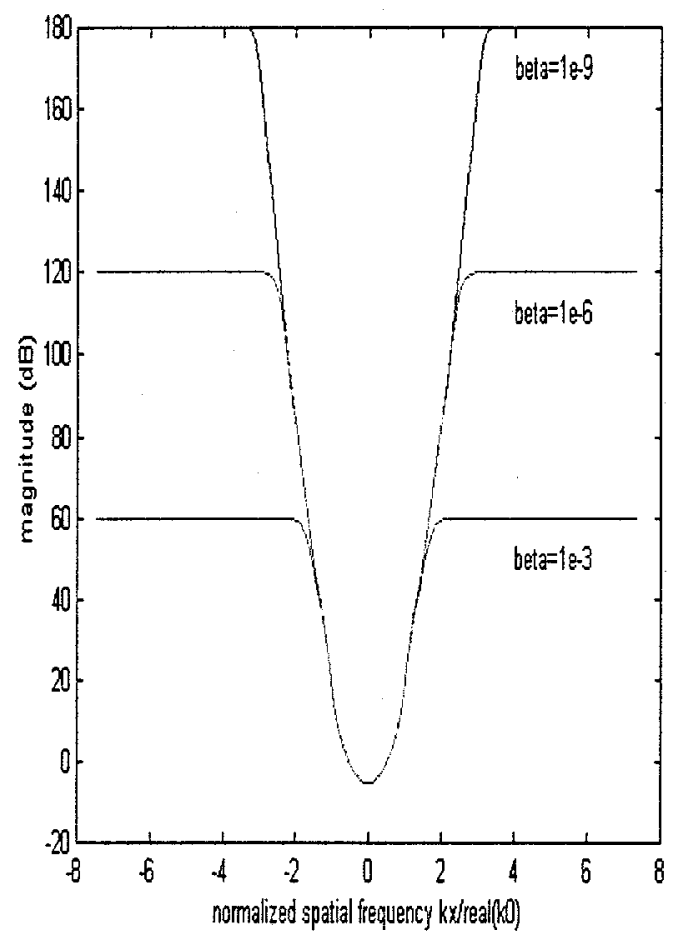

Figure 3: Magnitude of the filter vs. spatial frequency $K_{x}$ and $\beta=10^{-3}, 10^{-6}, 10^{-9}$ for a single frequency simulation with $\lambda=1.5 \mathrm{~m}$. 


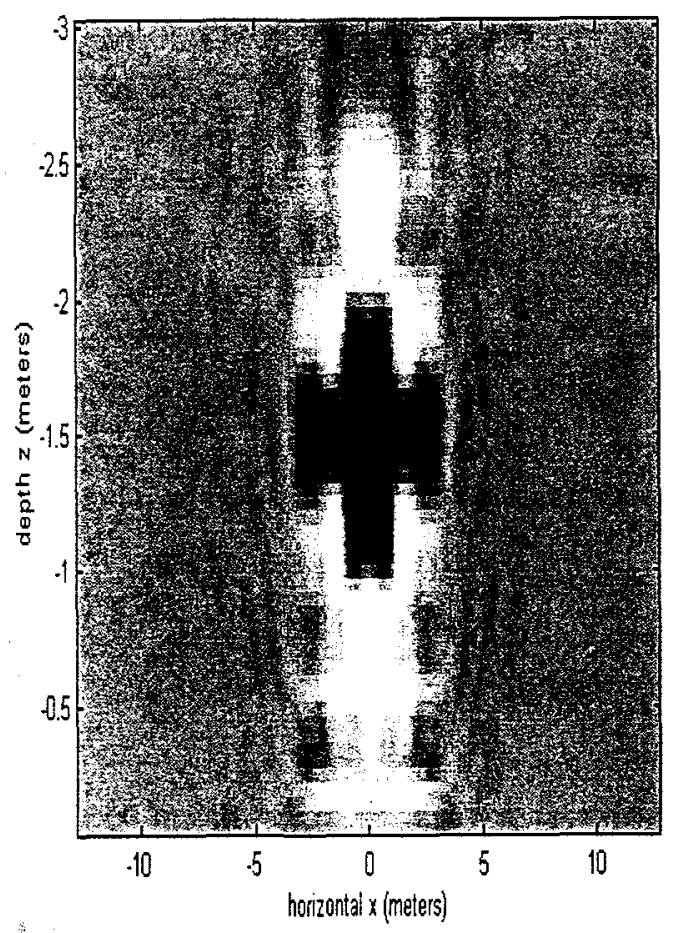

Figure 4: Pseudoinverse reconstruction based on a single excitation frequency corresponding to $\lambda=1.5$ meters. The penetration depth in the soil is 3 meters. 


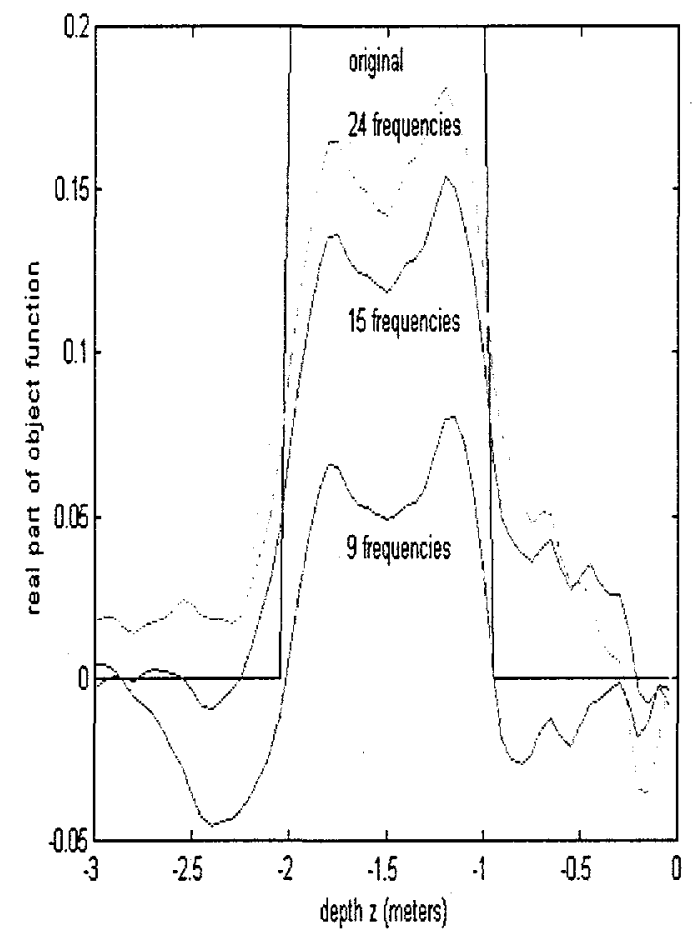

Figure 5: Cross sections at $x=0$ for Pseudoinverse reconstructions computed from 9, 15, and 24 frequencies corresponding to $0.375 m<\lambda<3.375 m$. 


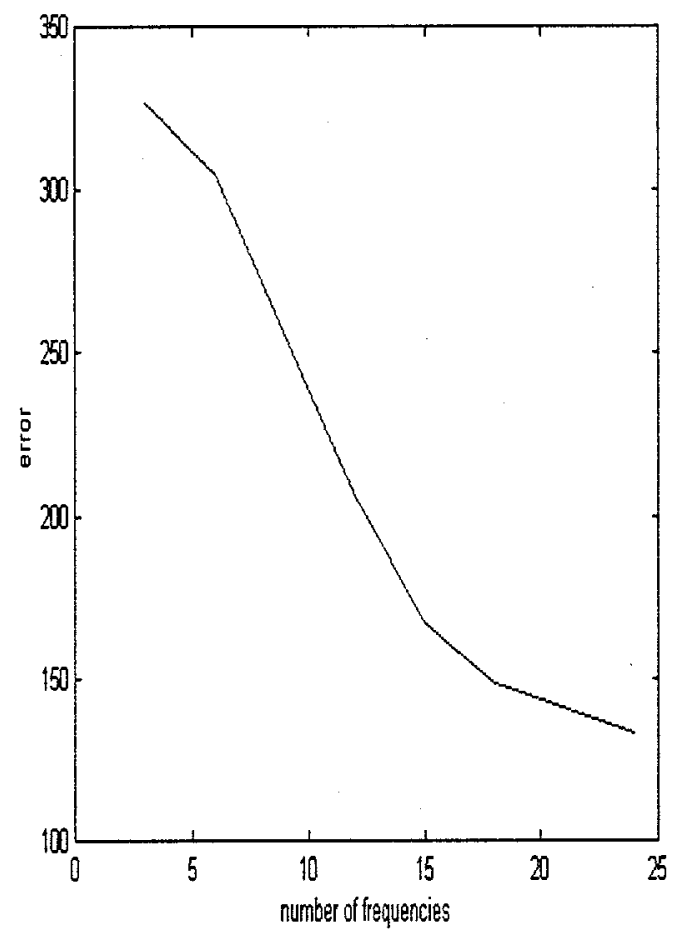

Figure 6: error $=\int d^{2} r\left|O(\mathbf{r})-\hat{O}_{\beta}(\mathbf{r})\right|^{2}$ vs. number of frequencies used in the reconstruction. Frequencies correspond to the range $0.375 m<\lambda<3.375 m$. 


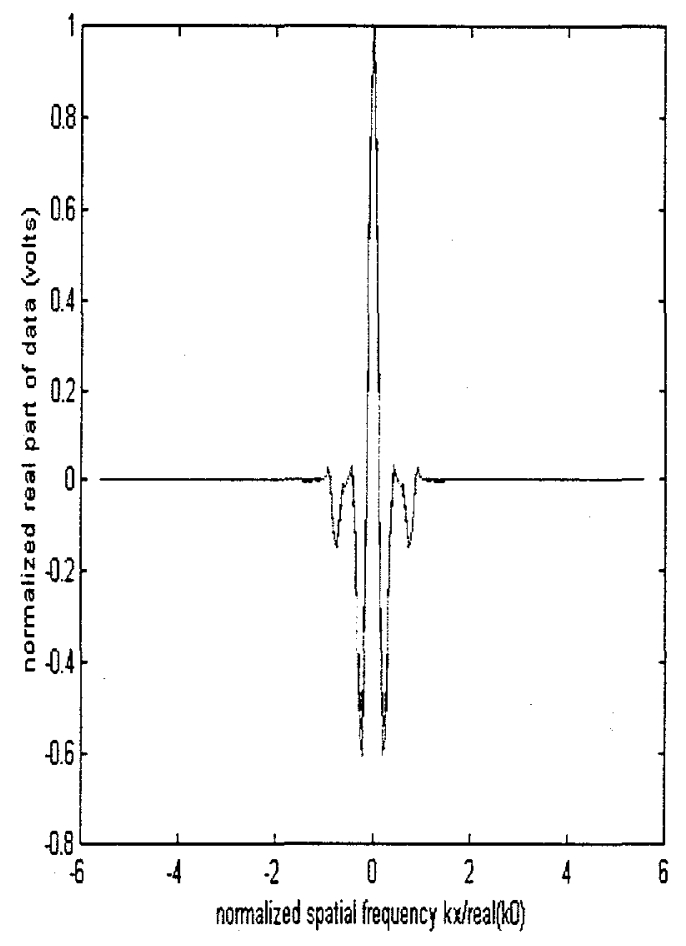

Figure 7: Real part of the data $\tilde{V}\left(\omega_{n} ; K_{x}\right)$ corresponding to $\lambda=1.125 \mathrm{~m}$ as computed from Eq.(54) on both the original object of Fig.2 and the reconstruction of Fig.5 computed with 9 frequencies.

scale shown), we conclude that the reconstruction in Fig. 5 is consistent with the data, as expected. 


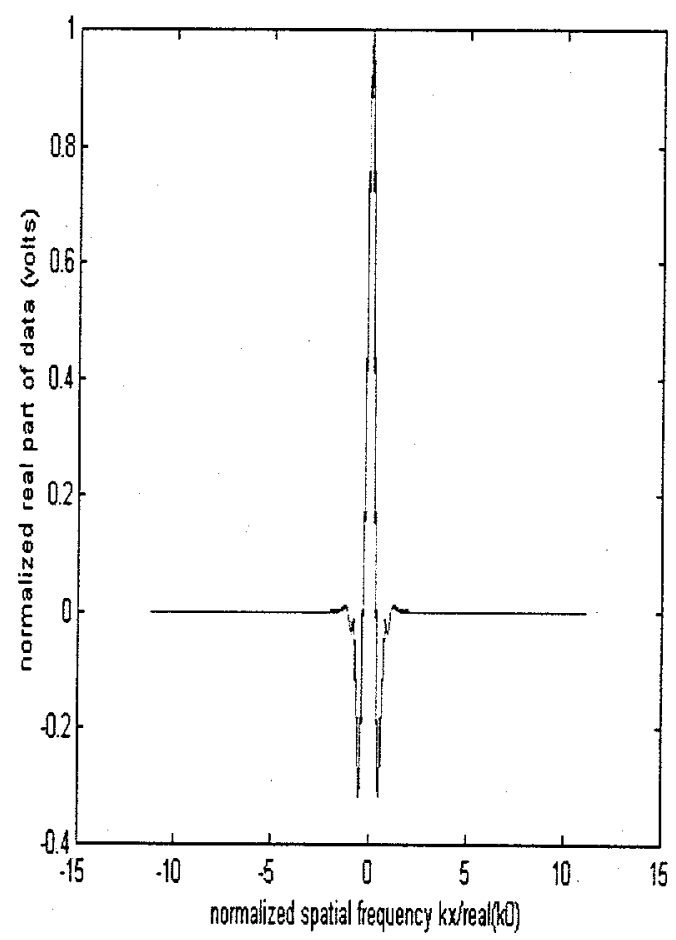

Figure 8: The same plot as Fig.8a, for data from the frequency corresponding to $\lambda=2.25 \mathrm{~m}$. 


\section{Summary and Conclusions}

In this report we derived a direct, non-iterative, inversion formula for multi-monostatic GPR data which was tested successfully on synthetic radar data. To solve this problem we cast the forward scattering relation in a mathematical operator framework, allowing computationally efficient, analytical expressions to be found for both the adjoint and pseudo inverse operators. This type of approach has been used with similar success in other tomographic imaging applications [4], and we anticipate future uses as well.

We mentioned above that significant noise will be inevitable in realistic GPR imaging applications. In preliminary studies, we have verified that our algorithm is robust in the presence of both additive noise at receivers, and noise due to scattering from ground clutter. In these noisy simulations, the results were quite sensitive to the value of the regularization parameter $\beta$, as expected. In a future publication we plan to present the results of these noisy simulations, as well as results using experimental GPR data.

The work carried out in this project is now (1999) being applied to GPR imaging in both DOE and commercial applications. A new project is currently under consideration by the DOE to image low level nuclear waste in Idaho and an on-going ARL project is employing these algorithms in problems of mine detection. In the commercial world a company (Witten Technologies) is investigating the use of these algorithms to located buried cables and utilities from multi-monostatic surveys. 


\section{References}

[1] Bertero M, DeMol C and Viano G A 1980 The stability of inverse problems Inverse Scattering Problems in Optics: Topics in Current Physics 20 ed Baltes H P (SpringerVerlag) $161-214$

[2] Bertero M 1988 Linear inverse and ill-posed problems Advances in Electronics and Electron Physics 75 ed Hawkes P W (New York: Academic) 1-121

[3] Chew W C 1990 Waves and Fields in Inhomogeneous Media (IEEE Press)

[4] Deming R W and Devaney A J 1995 A filtered backpropagation algorithm for GPR $J$. Environmental and Eng. Geophysics 0 113-23

[5] Devaney A J and Sherman G C 1973 Plane-wave representations for scalar wave fields SIAM Review 15 765-86

[6] Devaney A J 1983 Inverse source and scattering problems in ultrasonics IEEE Trans. on Sonics and Ultrasonics SU-30 355-64

[7] Devaney A J 1984 Geophysical diffraction tomography IEEE Trans. on Geosci. and Remote Sensing GE-22 3-13

[8] Devaney A J and Zhang D 1991 Geophysical diffraction tomography in a layered background Wave Motion 14 243-65

[9] Goodman J W 1968 Introduction to Fourier Optics (New York: McGraw-Hill)

[10] Hager W W 1988 Applied Numerical Linear Algebra (New Jersey: Prentice-Hall)

[11] Ishimaru A 1991 Electromagnetic Wave Propagation, Radiation, and Scattering (New Jersey: Prentice-Hall)

[12] Jain A K 1986 Fundamentals of Digital Image Processing (New Jersey: Prentice-Hall)

[13] Kerns D M 1981 Plane-Wave Scattering-Matrix Theory of Antennas and AntennaAntenna Interactions National Bureau of Standards Monograph 162

[14] Ladas K T and Devaney A J 1993 Application of an ART algorithm in an experimental study of ultrasonic diffraction tomography Ultrasonic Imaging 15 48-58

[15] Maleki M H and Devaney A J 1993 Phase-retrieval and intensity-only reconstruction algorithms for optical diffraction tomography J.Opt. Soc. Am. A 10 1-7 
[16] Morse P M and Feshbach H 1953 Methods of Mathematical Physics vols. I and II (New York: McGraw-Hill)

[17] Natterer F 1986 The Mathematics of Computerized Tomography (New York: Wiley)

[18] Noble B and Daniel J W 1988 Applied Linear Algebra (New Jersey: Prentice Hall)

[19] Norton S J and Linzer M 1981 Ultrasonic reflectivity imaging in three dimensions: exact inverse scattering solutions for plane, cylindrical, and spherical apertures IEEE Trans. Biomed. Eng. BME-28 202-20

[20] Pratt R G and Worthington M H 1988 The application of diffraction tomography to cross-hole seismic data Geophysics 53 1284-94

[21] Press W H, Teukolsky S A, Vetterling W T and Flannery B P 1992 Numerical Recipes in FORTRAN: the Art of Scientific Computing second edition (Cambridge University Press)

[22] Schatzberg A and Devaney A J 1992 Super-resolution in diffraction tomography Inverse Problems 8 149-64

[23] Schatzberg A, Devaney A J and Deming R W 1995 Maximum likelihood estimation of target location in acoustic and electromagnetic imaging Expanded Abstracts of the 65th Annual Meeting of the Society of Exploration Geophysicists (Houston)

[24] Slaney M, Kak A C and Larsen L E 1984 Limitations of imaging with first order diffraction tomography IEEE trans. Microwave Theory Tech. MTT-32 860-73

[25] Tarantola A 1984 Linearized inversion of seismic reflection data Geophys. Prosp. 32 998-1015

[26] Witten A J and Molyneux J E 1992 Geophysical diffraction tomography: validity and implementation Geophysical Inversion eds Bednar J B, Lines L R, Stolt R H and Weglein A B (SIAM) 354-69

[27] Witten A J, Molyneux J E and Nyquist J E 1994 Ground penetrating radar tomography: algorithms and case studies IEEE Trans. Geosci. and Remote Sensing 32 461-7

[28] Wu R and Toksoz M N 1987 Diffraction tomography and multisource holography applied to seismic imaging Geophysics 52 11-25

[29] Wu R, Araujo F V and Huang L 1994 Multifrequency backscattering tomography for constant and vertically varying backgrounds Int. J. Imaging Systems and Tech. 5 7-21 